\title{
Evaluation of effect of number of electrodes in ERT sensors on image quality
}

DOI:

10.1109/IST.2013.6729655

Link to publication record in Manchester Research Explorer

\section{Citation for published version (APA):}

Ye, J., Wang, H., \& Yang, W. (2013). Evaluation of effect of number of electrodes in ERT sensors on image quality. In IST 2013 - 2013 IEEE International Conference on Imaging Systems and Techniques, Proceedings/IST IEEE Int. Conf. Imaging Syst. Tech., Proc. (pp. 19-24). IEEE. https://doi.org/10.1109/IST.2013.6729655

\section{Published in:}

IST 2013 - 2013 IEEE International Conference on Imaging Systems and Techniques, Proceedings||IST - IEEE Int. Conf. Imaging Syst. Tech., Proc.

\section{Citing this paper}

Please note that where the full-text provided on Manchester Research Explorer is the Author Accepted Manuscript or Proof version this may differ from the final Published version. If citing, it is advised that you check and use the publisher's definitive version.

\section{General rights}

Copyright and moral rights for the publications made accessible in the Research Explorer are retained by the authors and/or other copyright owners and it is a condition of accessing publications that users recognise and abide by the legal requirements associated with these rights.

\section{Takedown policy}

If you believe that this document breaches copyright please refer to the University of Manchester's Takedown Procedures [http://man.ac.uk/04Y6Bo] or contact uml.scholarlycommunications@manchester.ac.uk providing relevant details, so we can investigate your claim.

\section{OPEN ACCESS}




\section{Evaluation of Effect of Number of Electrodes in ERT Sensors on Image Quality}

\author{
Jiamin Ye, Haigang Wang \\ Institute of Engineering Thermophysics \\ Chinese Academy of Sciences \\ Beijing 100190, China \\ yejiamin@iet.cn, wanghaigang@iet.cn
}

\author{
Wuqiang Yang \\ School of Electrical and Electronic Engineering \\ The University of Manchester \\ Manchester M13 9PL, UK \\ w.yang@manchester.ac.uk
}

\begin{abstract}
It is assumed that the spatial resolution of the reconstructed images with an electrical resistance tomography (ERT) system can be improved by increasing the number of measurement electrodes. In this paper, the issues with the number of electrodes will be discussed. To analyze the effect of the number of electrodes on the quality of reconstructed images, ERT sensors with different number of electrodes-8, 12, 16, 20, 24, 32, 36, 40 and 48 - are investigated. With the adjacent and opposite strategies, the voltages between different electrode pairs are calculated for some typical conductivity distributions with different contrast in conductivity using a finite element method. The obtained voltage data are then used to reconstruct images using the projected Landweber iteration algorithm. In the meanwhile, the impact of meshes in the inverse problem solver on image quality is also discussed. The sensitivity distributions for all cases are analyzed utilizing singular value decomposition (SVD). The analytical results are in agreement with the simulation results.
\end{abstract}

Keywords—resistance sensor; electrical resistance tomography; image reconstruction

\section{INTRODUCTION}

As a process tomography (PT) technique, electrical resistance tomography (ERT) is particularly useful for visualizing the conductive property and distributions of processes. It has been widely used in industrial [1] and medical [2] applications. Wang et al [2] studied the distribution of the sensing field affected by the configuration of the compound electrodes used in the medical EIT system and established a finite element model with the condition of the coercive equipotential nodes on the boundary of an object. Cao et al [3] designed an optimized square sensor for electrical impedance tomography in order to obtain maximum information in the sense of Shannon information entropy. Cui et al [4] studied an integrated electrical capacitance tomography (ECT)/ERT dual modality sensor. He et al [5] analyzed the electrical impedance tomography sensitive field based on multi-terminal network. Adler et al [6] developed a method to measure the information content and discussed important issues in electrical impedance tomography (EIT), such as distinguishability limits, optimal measurement schemes and data fusion. Polydorides and

The authors would like to thank the National Natural Foundation of China (No. 61072001) to support this work.
McCann [7] examined the impacts of the singular values of a sensitivity matrix on the spatial resolution of the reconstructed images and developed a system based on electrode segmentation, providing sensitivity matrices with improved characteristics. Tang et al [8] analyzed the effect of the number of electrodes and the number of conductivity basis functions on image reconstruction results in EIT, but did not consider the influence of measurement strategy and conductivity contrast.

Literature review shows that the effect of the number of electrodes on the image quality with ERT sensors has not been systematically discussed. A common assumption about the ECT or ERT sensors is that more electrodes would result in higher spatial resolution of the reconstructed images. While Peng et al. [9] investigated the effect of the number of electrodes in ECT sensors on image quality and pointed out that 12-electrode ECT sensors are recommended for most applications, rather than too many electrodes, the effect of the number of electrodes in ERT sensors on image quality should be discussed too.

In this paper, 9 ERT sensors with different number of electrodes, $8,12,16,20,24,32,36,40$ and 48 , are investigated by simulation, to study the effect of the number of electrodes on the quality of reconstructed images. For all ERT sensors used in this work, the impact of conductivity contrast, conductivity distribution, measurement strategy and meshes for finite element simulation on image quality is analyzed.

\section{SIMULATION ARRANGEMENTS}

\section{A. Simulation Arrangement}

While the adjacent excitation strategy [10] is the most popular for ERT, the opposite excitation strategy is used by some researchers [11]. In this research both the adjacent and opposite strategies are utilized. Fig. 1 shows an ERT sensor with $N$ electrodes. For the adjacent strategy, the total number of measured voltages is

$$
M=N \cdot(N-3) / 2
$$

For the opposite strategy, the total number of measurements in this strategy is

$$
M=N \cdot(N-4) / 2
$$




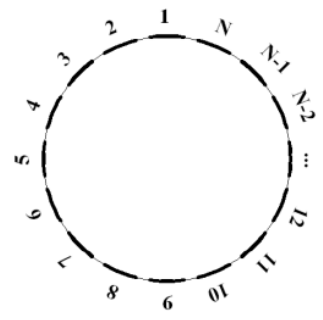

Fig. 1 ERT sensor with $N$ electrodes.

Pinheiro [12] pointed out that the optimal electrode covering ratio is $60 \%$ and $80 \%$, respectively, for the opposite strategy and adjacent strategy. To facilitate modeling and comparison, the electrode covering ratio is chosen to be $70 \%$ for both strategies in this work. The number of electrodes is changed from 8 to $12,16,20,24,32,36,40$ and 48 . Some typical conductivity distributions are considered. The materials with conductivities of $1 \mathrm{e}-10 \mathrm{~S} / \mathrm{m}$ and $0.02 \mathrm{~S} / \mathrm{m}$ are selected as the second phase against the background with a conductivity of 0.03 $\mathrm{S} / \mathrm{m}$. The normalized conductivity distributions are illustrated in Fig. 2.

To evaluate the image reconstruction quantitatively, the projected Landweber iteration is used throughout this work.To assess the quality of image reconstruction, the relative image error and the correlation coefficient between the true conductivity distributions and the reconstructed images are used as criteria. In the Landweber iteration, the steps of iteration play an important role. In this research, the iteration process will be stopped when the image error becomes larger and the correlation coefficient becomes smaller than that with the previous iteration step to avoid the local minima.

For forward problem modeling, the sensing domain is divided into sufficiently fine mesh so that the voltage measurements are not changed if the number of elements is further increased. The sensing area is then divided into $60 \times 60$, $30 \times 30$, and $20 \times 20$ grids for solving the inverse problem, with 2828, 716 and 316 pixels, respectively.

The data used in simulation are added $50 \mathrm{~dB}$ Gaussian noise, the magnitude of which is typical regarding the signalto-noise ratio (SNR) in EIT. Modeling and image reconstruction were carried out using COMSOL Multiphysics $^{\mathrm{TM}}$ and MatLab ${ }^{\mathrm{TM}}$.

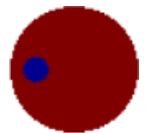

(a)

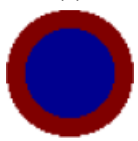

(e)

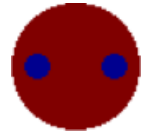

(b)

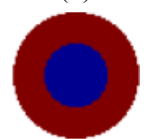

(f)

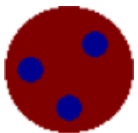

(c)

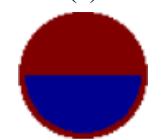

(g)

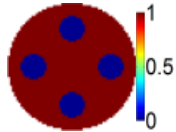

(d)

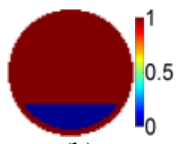

(h)
Fig. 2 Real conductivity distributions. (a) Single bar (b1). (b) Two bars (b2). (c) Three bars (b3). (d) Four bars (b4). (e) Annular distribution 1 (a1). (f) Annular distribution 2 (a2). (g) Stratified distribution 1 (s1). (h) Stratified distribution 2 (s2).

\section{RESULTS AND DISCUSSION}

\section{A. With a Mesh of $60 \times 60$ Grids}

This subsection gives the results of image reconstruction using the models with $60 \times 60$ grids.

\section{1) With a conductivity of the second phase of 1e-10 S/m}

Fig. 3 shows some images reconstructed by the Landweber iteration for 8 different distributions, b1, b2, b3, b4, a1, a2, s1 and $\mathrm{s} 2$ (see Fig. 2), with the adjacent strategy. The conductivity of the second phase is $1 \mathrm{e}-10 \mathrm{~S} / \mathrm{m}$. Fig. 4 illustrates the image error and the correlation coefficient. From the reconstructed images shown in Fig. 3, it can be seen that the quality of most images reconstructed by the Landweber iteration is increased with the increase in the number of electrodes from 8 to 12,16 , 20 and 24. The images from the ERT sensors with 32, 36, 40 and 48 electrodes present similar qualities. It can be concluded from Fig. 4 that the 32-electrode ERT sensor can give the best image reconstruction for most distributions.

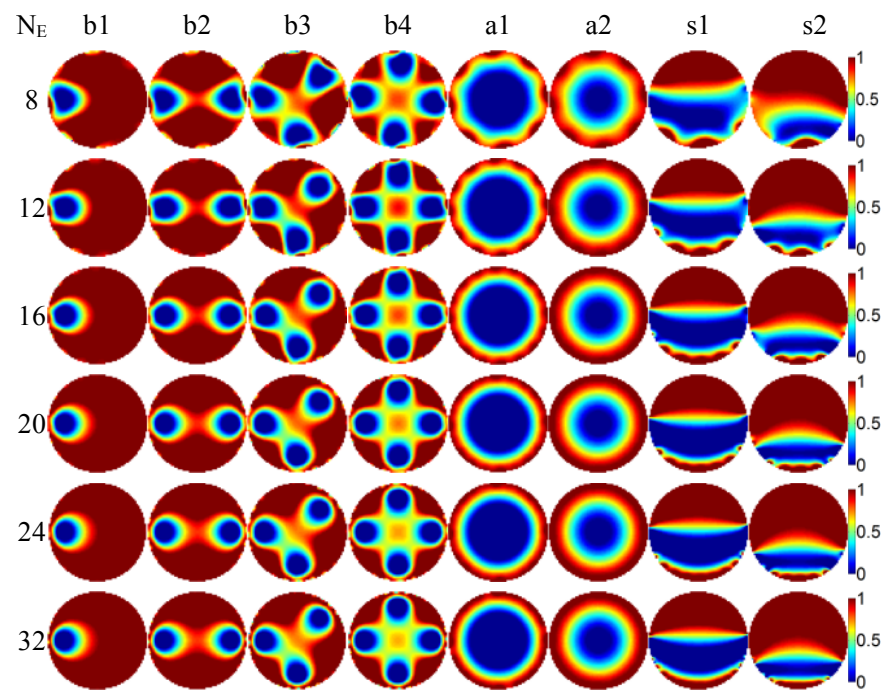

Fig. 3 Images reconstructed by Landweber iteration with adjacent strategy, where the conductivity of the second phase is $1 \mathrm{e}-10 \mathrm{~S} / \mathrm{m}$ and the sensing domain is divided into $60 \times 60$ grids.

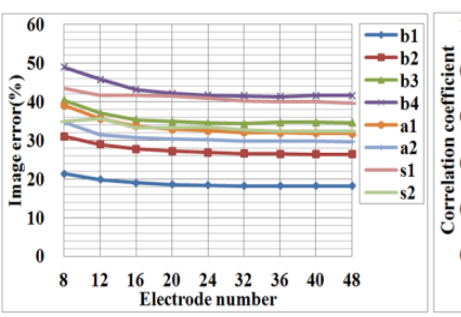

(a)

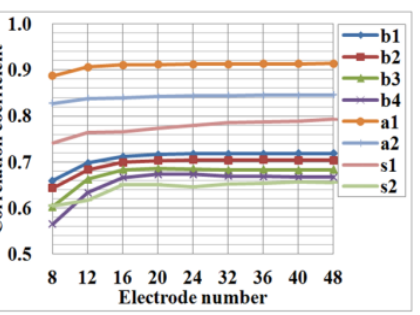

(b)
Fig. 4 Image error and correlation coefficient with adjacent strategy, where the conductivity of the second phase is $1 \mathrm{e}-10 \mathrm{~S} / \mathrm{m}$ and the sensing domain is divided into $60 \times 60$ grids. 


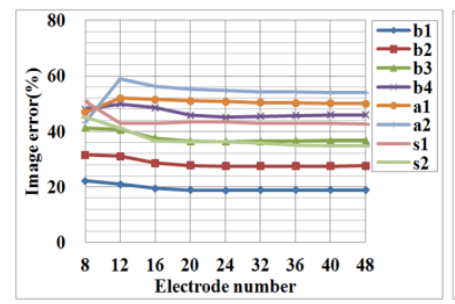

(a)

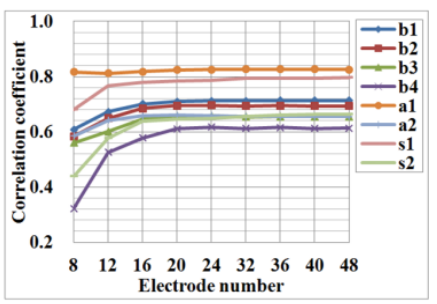

(b)
Fig. 5 Image error and correlation coefficient with opposite strategy, where the conductivity of the second phase is $1 \mathrm{e}-10 \mathrm{~S} / \mathrm{m}$ and the sensing domain is divided into $60 \times 60$ grids.

Fig. 5 illustrates the quantitative results including the image error and the correlation coefficient reconstructed by the Landweber iteration, with the opposite strategy. The conductivity of the second phase is $1 \mathrm{e}-10 \mathrm{~S} / \mathrm{m}$. It can be found from Fig. 5 that the 32-electrode ERT sensor can also give the best image reconstruction for most conductivity distributions.

2) With a conductivity of the second phase of $0.02 \mathrm{~S} / \mathrm{m}$

In this test, the conductivity of the second phase is set to be $0.02 \mathrm{~S} / \mathrm{m}$ and both the adjacent strategy and the opposite strategy are used. Similar images to those shown in Fig. 3 are

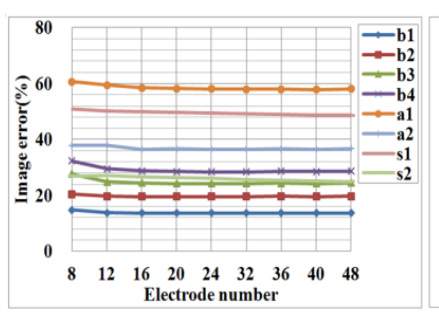

(a)

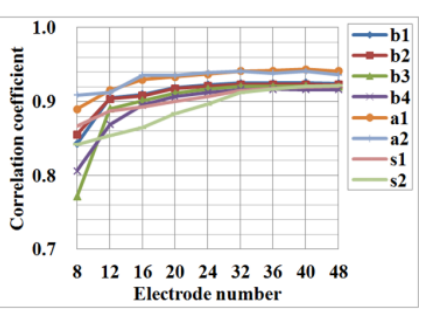

(b)
Fig. 6 Image error and correlation coefficient with adjacent strategy, where the conductivity of the second phase is $0.02 \mathrm{~S} / \mathrm{m}$ and the sensing domain is divided into $60 \times 60$ grids.

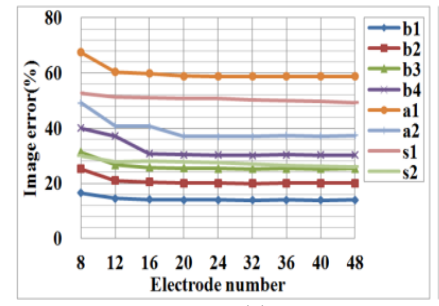

(a)

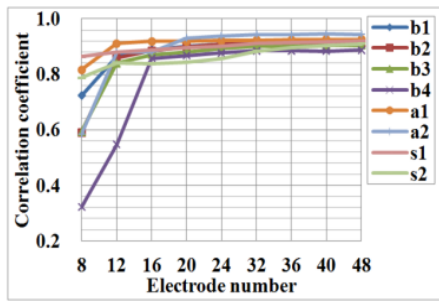

(b)
Fig. 7 Image error and correlation coefficient with opposite strategy, where the conductivity of the second phase is $0.02 \mathrm{~S} / \mathrm{m}$ and the sensing domain is divided into $60 \times 60$ grids. obtained by the Landweber iteration. Fig. 6 illustrates the quantitative results including the image error and the correlation coefficient with the adjacent strategy. It can be seen from the simulation results that the 32-electrode ERT sensor can also give the best result for most distributions. Fig. 7 illustrates the related quantitative results including the image error and the correlation coefficient with the opposite strategy. The image error and correlation coefficient illustrated in Fig. 7 become almost constant when the number of electrodes is more than 32 . It can be concluded from the simulation results that the 32electrode ERT sensor can give the best image reconstruction for most conductivity distributions.

\section{B. With a Mesh of $30 \times 30$ Grids}

For the distributions with conductivities of the second phase of $1 \mathrm{e}-10 \mathrm{~S} / \mathrm{m}$ and $0.02 \mathrm{~S} / \mathrm{m}$, images are reconstructed by the Landweber iteration. Because of the similarity to those shown in Fig. 3, the images are not shown. Fig. 8 illustrates the relevant quantitative results including the image error and the correlation coefficient with the sensing domain being divided into $30 \times 30$ grids. It can be seen that the 24 -electrode sensor can give the best image reconstruction for the bar distributions and the annular distributions. In comparison, the ERT sensor with 40 electrodes can give the best result for the stratified distributions.

\section{With a Mesh of $20 \times 20$ Grids}

With a mesh of $20 \times 20$ grids, images are reconstructed by the Landweber iteration for the distributions with conductivities of the second phase of $1 \mathrm{e}-10 \mathrm{~S} / \mathrm{m}$ and $0.02 \mathrm{~S} / \mathrm{m}$. Fig. 9 illustrates the quantitative results including the image error and the correlation coefficient.

It has been noted that the images reconstructed from the 48electrode ERT sensor for the distributions with the second phase with conductivity of $1 \mathrm{e}-10 \mathrm{~S} / \mathrm{m}$ have some artifacts, although the quantitative results for several distributions are better than that obtained from other sensors. For the distributions with the second phase with conductivity of $0.02 \mathrm{~S} / \mathrm{m}$, the reconstructed images from the 48-electrode ERT sensor suffer from serious distortions, especially for the annular distributions and stratified distributions. With consideration of the above analysis and the quantitative results, it can be concluded that the 40-electrode ERT sensor can give the best image reconstruction for most models. TABLE I summarizes the optimal number of electrodes under different conditions.

\section{Spectral Analysis using Singular Value Decomposition}

In image reconstruction for ERT, the sensitivity matrix is crucial because it presents the relationship between the measurements and the conductivity distributions. To explain the results of comparative studies specified in TABLE I, singular value decomposition (SVD) is applied in the spectral analysis. 


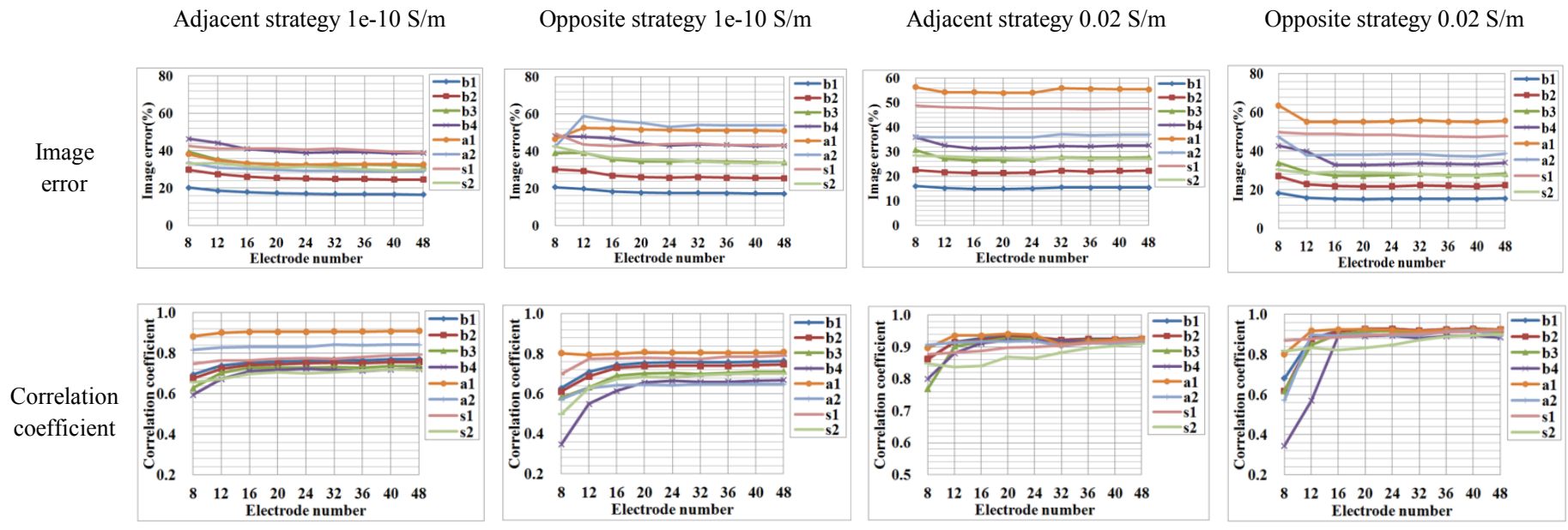

Fig. 8 Image error and correlation coefficient with Landweber iteration with different strategies and different conductivity of the second phase. The sensing domain is divided into $30 \times 30$ grids.
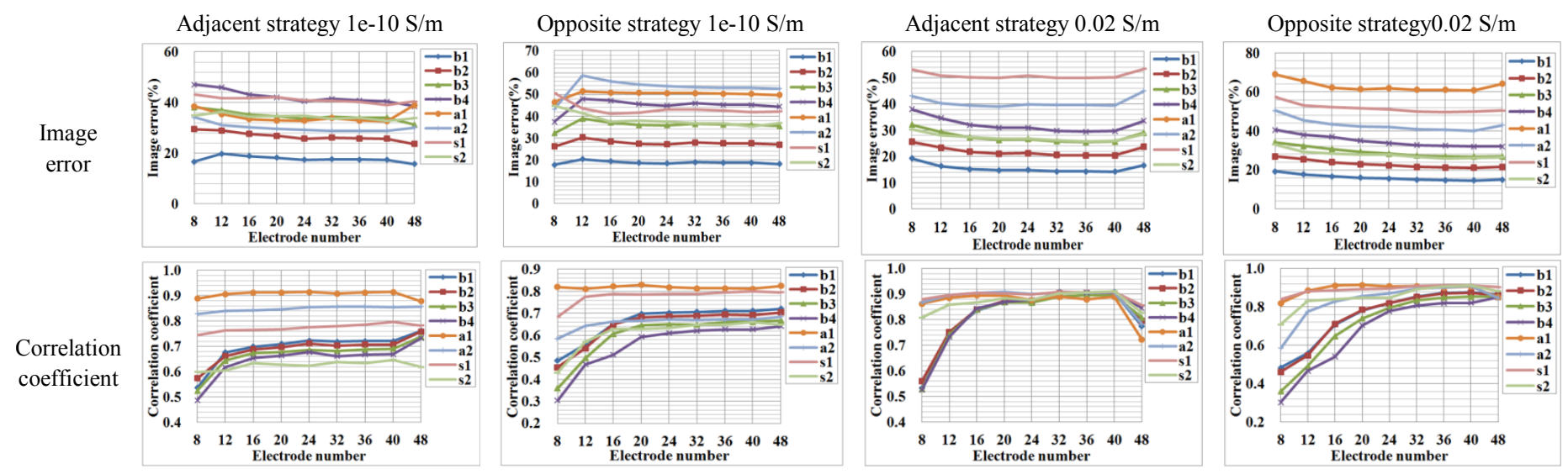

Fig. 9 Image error and correlation coefficient with Landweber iteration with different strategies and different conductivity of the second phase. The sensing domain is divided into $20 \times 20$ grids.

In Fig. 10, the magnitudes of the singular values for the models with different number of electrodes are compared. The legends in Fig. 10 consist of the measurement strategy, the mesh and the number of electrodes. For example, A-60-8e represents that the measurement strategy is the adjacent strategy, the sensing domain is divided into $60 \times 60$ grids and the number of electrodes is 8. Similarly, O-30-12e means that the opposite strategy, $30 \times 30$ grids and 12 -electrode sensor are used in the study. Some magnitudes of the singular values calculated by applying the opposite strategy, which are less than $10^{-10}$, have little effect on the reconstruction. This implies that the number of independent measurements is less than the number of measurements calculated by (2).

With $60 \times 60$ grids, the number of the singular values, which are nearly to zero, increases gradually with the number of electrodes. For the other two meshes, the number of measurements becomes more than the number of pixels in the sensing domain when the number of electrodes is more than a certain value. To be more specific, this phenomenon appears if the number of electrodes is equal to or more than 32 and 40 with $20 \times 20$ grids and $30 \times 30$ grids, respectively.

As can be seen from Fig. 10, with $60 \times 60$ grids, the unique singular values tend to have some small magnitudes, which are less than $10^{-10}$ if the number of electrodes is more than 24 . The number of the singular values with small magnitudes increases rapidly with the number of electrodes. The singular values with small magnitudes make a contribution to the reconstruction for finer details. However, too many small singular values will reduce the stability of measurements and increase the susceptibility to noise. Therefore, the sensitivity matrices obtained from the model with 32 electrodes using either the adjacent strategy or the opposite strategy can provide adequate information for image reconstruction for the coarse shape and fine details of the object. 
$60 \times 60$ grids
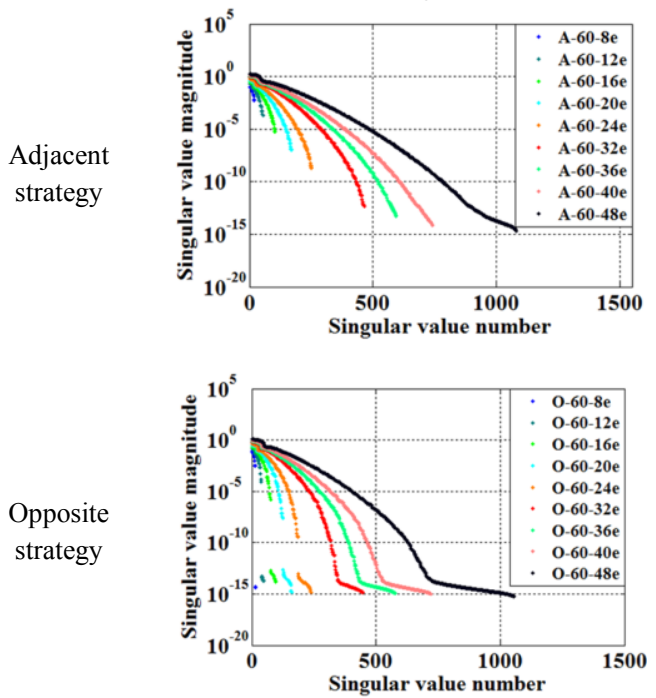

$30 \times 30$ grids
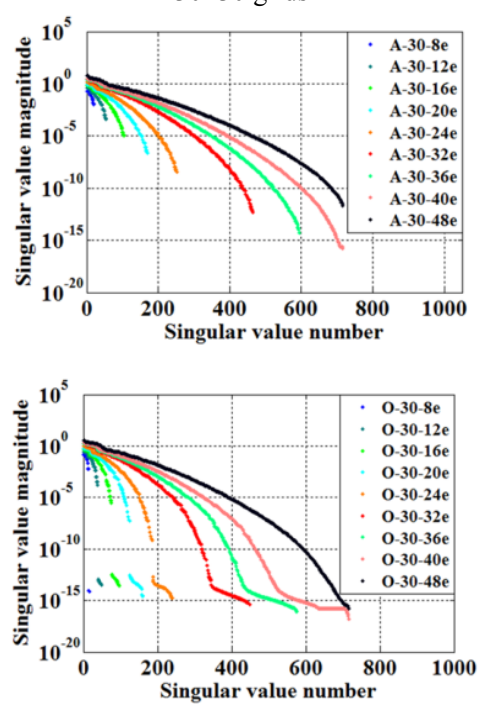

$20 \times 20$ grids
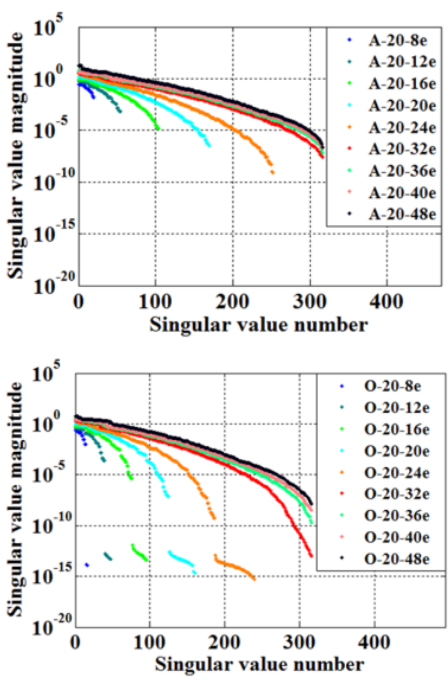

Fig. 10 Spectral analysis for the models with different number of electrodes.

TABLE I SUMMARY OF OPTIMAL NUMBER OF ELECTRODES UNDER DIFFERENT CONDITIONS.

\begin{tabular}{|c|c|c|c|c|c|}
\hline \multirow{2}{*}{$\begin{array}{l}\text { Conductivity } \\
\text { of the } \\
\text { second } \\
\text { phase }(\mathrm{S} / \mathrm{m})\end{array}$} & \multirow[b]{2}{*}{ Distribution } & \multirow[b]{2}{*}{$\begin{array}{c}\text { Measurement } \\
\text { strategy }\end{array}$} & \multicolumn{3}{|c|}{ Mesh } \\
\hline & & & $\begin{array}{l}60 \times 60 \\
\text { grids }\end{array}$ & $\begin{array}{c}30 \times 30 \\
\text { grids }\end{array}$ & $\begin{array}{c}20 \times 20 \\
\text { grids }\end{array}$ \\
\hline \multirow{4}{*}{$1 \mathrm{e}-10$} & \multirow{2}{*}{$\begin{array}{c}\mathrm{b} 1, \mathrm{~b} 2, \mathrm{~b} 3, \mathrm{~b} 4, \\
\mathrm{a} 1, \mathrm{a} 2\end{array}$} & Adjacent & \multirow{2}{*}{32} & \multirow{2}{*}{24} & \multirow{2}{*}{40} \\
\hline & & Opposite & & & \\
\hline & \multirow{2}{*}{$\mathrm{s} 1, \mathrm{~s} 2$} & Adjacent & \multirow{2}{*}{32} & \multirow{2}{*}{40} & \multirow{2}{*}{40} \\
\hline & & Opposite & & & \\
\hline \multirow{3}{*}{0.02} & \multirow{2}{*}{$\begin{array}{c}\mathrm{b} 1, \mathrm{~b} 2, \mathrm{~b} 3, \mathrm{~b} 4 \\
\mathrm{a} 1, \mathrm{a} 2\end{array}$} & Adjacent & \multirow{2}{*}{32} & \multirow{2}{*}{24} & \multirow{2}{*}{40} \\
\hline & & Opposite & & & \\
\hline & $\mathrm{s} 1, \mathrm{~s} 2$ & Adjacent & 32 & 40 & 40 \\
\hline
\end{tabular}

For the meshing method with $30 \times 30$ grids, the density of the grid is decreased in comparison to the mesh with $60 \times 60$ grids. This means that it requires less singular values with small magnitudes to reconstruct the details of the image. Therefore, the information provided by the 24-electrode sensor is sufficient to obtain a good image reconstruction for most distributions except for the stratified distributions, which have finer geometries. From the simulation results it can be seen that the image quality for most distributions with the second phase with conductivity of $0.02 \mathrm{~S} / \mathrm{m}$ decreases significantly when the number of electrodes is changed from 24 to 32 . The likely reason is that the reconstruction for the distributions with small conductivity contrast is more sensitive to the singular values with small magnitudes when the sensing domain is divided into $30 \times 30$ grids.
With $20 \times 20$ grids, the distribution of the magnitudes of singular values is almost the same if the number of electrodes is more than 40 . In the meanwhile, the magnitudes of the singular values are greater than $10^{-10}$, indicating that the singular components are stable under this condition. Although the magnitudes of the singular values from the 24- or 32-electrode ERT sensors are also greater than $10^{-10}$, the spectrum from the 40 electrode ERT sensor is smoother. The steeper the spectrum is, the worse the ill-posed condition in the image reconstruction is. Consequently, the ERT sensor with 40 electrodes can give the best reconstruction for most cases.

\section{CONCLUSIONS}

By considering different contrast in conductivity, conductivity distributions, measurement strategies and meshes, the effects of the number of electrodes in an ERT sensor on image reconstruction with 9 ERT sensors with different number of electrodes, 8, 12, 16, 20, 24, 32, 36, 40 and 48 are discussed.

Simulation results and SVD analysis show that the best image reconstruction for the models with fine meshes in the inverse problem solver can be obtained with 32 electrodes, regardless of the contrast in conductivity, conductivity distribution, using the Landweber iteration algorithm. The fine meshes, e.g. $60 \times 60$ grids, mean that the number of pixels in the sensing domain is more than the number of voltage measurements for the ERT sensors with different number of electrodes.

With a reduced number of pixels in the sensing domain but still keeping it more than the number of voltage measurements for majority of the sensors, e.g. $30 \times 30$ grids, the 24 -elelctrode sensor can reconstruct the best images for most distributions except for finer structures.

For the coarse meshes, e.g. $20 \times 20$ grids, where the number of pixels in the sensing domain is mostly less than the number 
of voltage measurements with the increase in the number of electrodes, the 40-electrode ERT sensor can give the best reconstruction for almost all of the cases.

\section{REFERENCES}

[1] J. Nowakowski, E. Hammer, D. Sankowski, D. Styra, R. Wajman, R. Banasiak and A.Romanowski, "New concept of ECT/ERT/GRT tomography for multi phase flow measurements," Automatyka ( o $^{3}$ rocznik $A G H)$, vol.14, pp. 741 - 748, 2010.

[2] H. X. Wang, C. Wang, and W. L. Yin, "Optimum design of the structure of the electrode for a medical EIT system," Measurement Science and Technology, vol.12, pp. 1021 - 1023, 2001

[3] Z. Cao, H. X. Wang and L. J. Xu, "Electrical impedance tomography with an optimized calculable square sensor," Review of Scientific Instruments, vol.79, pp. 103710 - 103710-7, 2008.

[4] Z. Q. Cui, H. X. Wang, Y. B. Xu and L. F. Zhang, "An integrated ECT/ERT dual modality sensor," In Instrumentation and Measurement Technology Conference, 2009, IEEE, Singapore, pp. 1434-1438, 2009.

[5] Y. B. He, H. X. Wang and D. Sankowski, "Analysis of electrical impedance tomography sensitive field based on multi-terminal network," Automatyka (pó3-rocznik AGH), vol.12, pp. 903 - 909, 2008.
[6] A. Adler, R. Youmaran and W. R. B. Lionheart, "A measure of the information content of EIT data," Physiological Measurement, vol.29, pp.S101-S109, 2008

[7] N. Polydorides and H. McCann, "Electrode configurations for improved spatial resolution in electrical impedance tomography," Measurement Science and Technology, vol.13, pp. 1862-1870, 2002.

[8] M. X. Tang, W.Wang, J. Wheeler, M. McCormick and X. Z. Dong, "The number of electrodes and basis functions in EIT image reconstruction," Physiological Measurement, vol.2, pp.129-140, 2002.

[9] L. H. Peng, J. M. Ye, G. Lu and W. Q. Yang, "Evaluation of effect of number of electrodes in ECT sensors on image quality," IEEE Sensors Journal, vol.12, pp. 1554-1565, 2012.

[10] A. D. Seagar, D. C. Barber and B. H. Brown, "Electrical impedance imaging," IEE Proceedings A, vol.134, pp.201-210, 1987.

[11] J. G. Webster, (ed.), Electrical Impedance Tomography, IOP: Bristol, 1990.

[12] P. A. T. Pinheiro, W. W. Loh and F. J. Dickin, "Optimal sized electrodes for electrical resistance tomography," Electronics Letter, vol.34, pp. 69-70, 1998. 